\title{
Musical Style Identification with $n$-Grams and Neural Networks
}

\author{
Pedro P. Cruz-Alcázar and María J. Castro-Bleda \\ ${ }^{1}$ Departamento de Informática de Sistemas y Computadores \\ ${ }^{2}$ Departamento de Sistemas Informáticos y Computación \\ Universidad Politécnica de Valencia \\ Valencia, Spain \\ ppcruz@disca.upv.es, mcastro@dsic.upv.es
}

\begin{abstract}
Musical Style Identification (MSI) aims to automatically classify music by style. It is being recently explored, mostly in the field of multimedia databases, with potential applications to content-based retrieval. But MSI may be also employed in other applications. We try to face up this challenge with two different methodologies: $n$-gram Models and Neural Networks. Very good results were obtained with $n$-grams in our previous research and we were willing to test how other Artificial Intelligence techniques performed with this task, so we began a preliminary study with Multilayer Perceptrons that is promising.
\end{abstract}

Keywords: Computer music, multimedia analysis, musical style identification, $n$-gram models, neural networks.

\section{Introduction}

Musical Style Identification (MSI) aims to automatically classify music by style. The MSI area is being recently (and not so much) explored, mostly in the field of multimedia databases, trying to improve content based retrieval by means of joining indexing by musical style to other suitable indexes. But other applications can be its use in the Musicology field (finding authors for anonymous pieces, which can be really hard for musicologists) or in Automatic Composition systems (as an evaluation function to be used in the search process performed by some of these systems). Some Pattern Recognition and AI-related techniques that have been employed so far are Hidden Markov Models [1], Self-Organising Maps and $k$-Nearest Neighbors [2], Neural Networks (NNs) [3] and Grammatical Inference (GI) 4. The use of GI techniques has been also widely studied by the paper's first author [567], being the best of all the $n$-grams models and achieving very good results with them, as will be presented in Section 6. This led us to test how other AI-techniques, like NNs, performed with this task and began a preliminary study with Multilayer Perceptrons (MLPs) that will be presented in this paper.

Focusing on the Music area, some issues had to be dealt with. Main features of a musical style are rhythm and melody. For the sake of conciseness, in what follows, we will use just the term "melody" for the combination of these two features. Our attention has focused on one-voice melodies, leaving polyphony 
for future works. The way music is represented implies the inclusion of more or less musical information, so, four music representations have been used in our study in order to asses their influence. Next sections are devoted to present the methodologies used in our classification experiments, $n$-gram models and MLPs.

\section{$2 \quad n$-Gram Models}

One of the most widely used models in Natural Language Processing is the $n$-grams one [8]. An $n$-gram is a sequence of symbols of length $n$, being the first $n-1$ of these the "context" of the $n$-th symbol. The size of $n$ can in theory be anything from 1 upwards. However, certain values are better than others at capturing the characteristics of the language. The larger the value of $n$, the more context is captured. Though it would seem useful to have a great $n$, it is not a case of the larger the better. As $n$ grows, it captures more context, but looses generality and learns the exact sequences in the corpus. There are two stages involved in using $n$-gram models: parameter estimation 8, where probabilities are assigned to $n$-length sequences found in a corpus, based on their frequency and the frequency of their context; and using these parameters for either evaluation or generation.

Even the largest corpora available will contain only a fraction of the possible $n$-grams. This requires the use of data smoothing techniques in order to provide better estimates of the more infrequent or unseen events. The most usual one consists in discounting some probability mass from the observed events to distribute it between the unseen ones. Also, if a given $n$-gram has not been observed in the training data, its probability can be estimated from the lower order model (the $(n-1)$-gram) and so on, using (if necessary) all the hierarchy of $(n-i)$-grams $[i=1 . . n]$ in a process known as backing-off [9]. Frequently, backing-off and discounting are combined according to the scheme devised by Katz 9]. This is the most popular smoothing method and is fairly well known as Back-off smoothing. This is the approach to data smoothing used in our experiments, where modeling with $n$-grams is performed using the CMU-Cambridge Statistical Language Modeling Toolkit (SLM-Toolkit) 10. It provides four possible discounting functions: Good-Turing, Linear, Absolute and Witten-Bell. All of them were tested in previous works [5]6] and Witten-Bell discounting [1] gave the best results, so it was chosen for our experiments.

\section{Multilayer Perceptrons}

Artificial NNs can be viewed as weighted directed graphs in which artificial neurons are nodes, and directed edges (with weights) are connections between neuron outputs and neuron inputs. Based on the connection pattern (architecture), neural networks can be grouped into two categories: feed-forward networks (with no loops) and recurrent networks (with feedback connections). Multilayer Perceptrons (MLPs) are the most common type of feed-forward networks and the neurons are organized into layers that have unidirectional connections between them (that is, an MLP consists of an input layer, one or more hidden 
layers, and an output layer of neurons) [1213. In this context, the (supervised) learning process can be viewed as the problem of updating connection weights from available training patterns so that the network can efficiently perform a specific task. Performance is improved over time by iteratively updating the weights in the network. This learning process can be efficiently performed with the backpropagation algorithm [12].

For classification purposes, the input layer holds the input samples (in this case, melodies), and the output layer is defined in accordance with the number of classes (musical styles). The values of the output layer can be seen as an approximation of the a posteriori probability of belonging to the associated class. Therefore, the output of the MLP is a function $g_{c}(d, \omega)$, where $c$ is the $c$-th output unit of the MLP with parameters $\omega$, given the input sample $d$, which estimates the a posterior probability $\operatorname{Pr}(c \mid d)$. Thus, for MLP classifiers the classification rule is as follows:

$$
c^{\star}=\underset{c}{\operatorname{argmax}} \operatorname{Pr}(c \mid d) \approx \underset{c}{\operatorname{argmax}} g_{c}(d, \omega) .
$$

The training of the MLPs was here carried out by using the neural network simulation software kit "SNNS: Stuttgart Neural Network Simulator" 14.

\title{
4 Musical Representations
}

Even though only duration and pitch of sounds (as main features of music) are used in this work, the way they are represented implies the inclusion of more or less musical information. The amount and/or meaning of this musical information can be crucial for the success in MSI. Many efforts have been done within the Computer Music research community in musical representation systems [15 16] but it is not clear that one system is always better than the others 15 17, being very dependent on the application and the recognition paradigm. In our previous works from the first author of this paper, a wide study of different representations was done 677.

In next subsections we present a selection of the pitch and duration representations that we considered more suitable for a preliminary study with Multilayer Perceptrons. Brief comments will be given for every representation, according to the advantages and disadvantages observed in previous works.

\subsection{Pitch Representation}

\begin{abstract}
Absolute. Pitch is most often represented either by the traditional pitch naming system (e.g. F\#4-G\#4-A4) or as absolute pitch (e.g. in MIDI: 66, 68, 69). It is the same representation as in musical scores, but may be insufficient for applications in tonal music. The main problem is that transpositions are not accounted for (e.g. the same pitch motive transposed in different samples or within the same one is considered as different motives).
\end{abstract}

Relative. A solution for the transposition problem is the use of the relative pitch between notes. That is, the interval (number of semitones) between two 
notes. There exists a somewhat 'peculiar' relationship between pitch strings and pitch interval strings. If one pitch interval in a string of pitch intervals is altered then all the succeeding notes are altered (transposed) [16. So, a change in a string of pitches and in a string of pitch intervals is not exactly the same thing. The latter is more significant and may be more 'dangerous', depending on the task being carried out. This effect appears clearly when musical style models built from samples in the relative representation are used to generate melodies (Automatic Composition), as we observed in our previous work in this area [7].

\subsection{Duration Representation}

In terms of the rhythmic component of musical strings, almost the same representations as for pitch can be applied. Moreover, it should be noted that the problems that arise with pitch representations (highlighted in the previous section) apply also for duration representations.

Absolute. This is a direct translation of the representation used in musical scores (e.g. whole note, half note, quarter note, and so on). It is the most commonly applied representation in musical string processing algorithms [16, maybe because it's easy to understand and straightforward to obtain.

Relative. According to our observations, listeners usually remember a rhythmic pattern as a relative sequence of durations that is independent of an absolute tempo. So, with absolute duration encoding, the same rhythmic pattern written with two different metrics will be considered as two different patterns. Representing rhythm as duration ratios can overcome augmentations or diminutions of a rhythmic pattern.

\subsection{Representations Encodings}

Being the $n$-gram models a Syntactic Pattern Recognition technique, it is usual to encode the training samples as symbol strings. In order to obtain the musical string, we have used numbers for pitch and duration representations, except for the Absolute duration representation, where symbols were used ( $r$ for whole note, $b$ for half note, $n$ for quarter note, and so on).

When using this representation with MLPs, numbers being proportional to the note lengths were assigned to each duration symbol. The experiments with MLPs were performed by using the following coding of the data: each pith or duration value was normalized to fit into the interval $[0,1]$. Moreover, the input layer of the MLPs were fixed to accept the longest training sample (shorter samples were centered along the input layer, filling with "rest notes" (zeros) the unused input units).

\section{Corpora}

During our research, we have worked with two different corpora that will be described in this section. Both are formed by melodies from different occidental 
musical styles, with 100 melodies for each style. Due to the lack of public domain musical corpora focused to this kind of study, the ones used have been compiled by the paper's first author. The compilation of such kind of corpora is not easy, and some tools were developed for automating the sample collecting. We chose, as musical source, audio files in MIDI format, and we had also to deal with some issues like preprocessing of polyphonic MIDI files, inexact duration of the notes, and so on, as discussed in 6]. Different criteria were used in the creation of each corpus, like musical style features and sample lengths, depending on the direction we wanted to focus our studies.

Corpus I. The first corpus is formed by 3 musical styles from different epochs. We collected 300 samples (100 per style) with a length ranging from 18 to 75 notes. That is, fragments from melodies, but not chosen at random. We picked up what is called in music, musical sentences, or fragments with musical significance. This resulted in 12062 running notes for this corpus. The first style was Gregorian (Middle Ages). As a second style, we used passages from the sacred music of J. S. Bach (Baroque). The third style consisted of passages from Scott Joplin's Ragtimes for piano (beginning of 20-th century).

Corpus II. After performing many experiments with the first corpus, we decided to create another one, mainly in order to increase the difficulty of the two Music Processing tasks we were dealing with. In order to make the classification task a little more realistic, the number of musical styles was increased by one, so in this corpus we have 4 styles. But, in addition, two musical styles were elected to be very close, stylistically speaking. These are two substyles from Celtic $\mathrm{Mu}-$ sic, the Celtic dances called Jigs and Reels. The other two styles are Gregorian, like in the Corpus I and Domenico Scarlatti's harpsichord sonatas, from the Baroque period. In this corpus, 400 samples were collected (100 per style). An important feature is that they are not melody fragments, but whole musical pieces, ranging from 61 to 1825 notes (220157 running notes). This allows us to study the influence from sample lengths in the style identification task.

\section{Experiments and Results}

The performed experiments with $n$-grams and MLPs are detailed in this section. Within the experiments, a 10-fold cross-validation has been used to measure the identification accuracy of the different techniques and coding schemes. The same partitions have been used with both $n$-grams and MLPs. The Average Classifying Error in identification for each style has been obtained, together with its corresponding 95\% confidence interval, and the best results for each technique are presented here.

\subsection{Experiments with $\boldsymbol{n}$-Grams}

In the experiments carried out using this technique, $n$-gram models (one per musical style) are inferred, studying different values of $n$. Test melodies (different from those seen in the inference process) are analyzed with all of the learned 
Table 1. Results with the $n$-gram models

\begin{tabular}{clccc}
\hline Codification encoding & \multicolumn{3}{c}{ Corpora } \\
Pitch & Duration & \multicolumn{2}{c}{ Corpus I } & \multicolumn{2}{c}{ Corpus II } \\
\hline Absolute & Absolute & $3.3[1.6,6.0]$ & 3.0 & {$[1.6,5.2]$} \\
Absolute & Relative & $5.0[2.8,8.1]$ & 6.0 & {$[3.9,8.8]$} \\
Relative & Absolute & $3.0[1.4,5.6]$ & $\mathbf{0 . 5}[\mathbf{0 . 0 6 , 1 . 8}]$ \\
Relative & Relative & $\mathbf{1 . 7}[\mathbf{0 . 5 , 3 . 8}]$ & 3.3 & {$[1.7,5.5]$} \\
\hline
\end{tabular}

models and their posterior probability is obtained. Their musical style is identified as the one from the model that gives the highest probability. The best results achieved with this technique are presented in Table 1 and they clearly show the influence of the musical representation. The results presented here are not so conclusive about the direction of this influence, but in previous works more musical representations were tested and the best results were obtained (in general) with the relative pitch representation. The results with $n$-grams are discussed in depth in [7].

\subsection{Experiments with MLPs}

In order to successfully use NNs as classifiers, several aspects have to be considered, such as the network topology, parameter setting of the training backpropagation algorithm and stopping criteria 121314. As commented before, the input layer of the MLPs were fixed to accept the longest training sample (shorter samples were centered along the input layer). Thus, 150-units input layer was used for Corpus I (the longest fragment from melody was composed by 75 notes and each note is represented by a "pitch, duration" pair). Corpus II was composed of whole musical pieces, ranging from 61 to 1825 notes. Such big variability in length is a serious inconvenience to work with fixed-dimensional input NNs, as MLPs are. Nevertheless, we desired to test the performance of MLPs with this corpus of melodies, using MLPs with one 3650 -units input layer for Corpus II.

One output unit is needed for each class; thus, 3 output units for Corpus I, and 4 output units for Corpus II, were used. Different number of hidden units were tested, from 20 to 150, and different values for the learning rate. To prevent over-fitting, a validation stopping criterion was followed. That is, $10 \%$ of the training data was randomly selected for validation purposes. Training was followed while the squared-error cost function (difference between desired output and actual output) was decreasing on the validation data. Random presentation of the training samples was used in the training process. The details of the experiments can be found in [18. The (best) results are summarized in Table 2 .

\subsection{Discussion}

We can see from results with $n$-grams shown in Table 1 that very low classification errors are obtained, being the best one a $1.7 \%$ for the Corpus I and 
Table 2. Results with Multilayer Perceptrons

\begin{tabular}{|c|c|c|c|}
\hline \multicolumn{2}{|c|}{ Codification encoding } & \multicolumn{2}{|c|}{ Corpora } \\
\hline Pitch & Duration & Corpus I & Corpus II \\
\hline Absolute & Absolute & {$[3.6,9.3$} & $23.3[19.2,27.7]$ \\
\hline Absolute & Relative & {$[2.1,6.9]$} & $38.7 \quad[33.9,43.7]$ \\
\hline Relative & Absolute & $21.3[16.8,26.4]$ & - \\
\hline Relative & Relative & $8.3[5.5,12.0]$ & $37.50 \quad[32.7,42.4]$ \\
\hline
\end{tabular}

an "outstanding" $0.5 \%$ for Corpus II (which means that only two samples out of 400 were misclassified). The best values for Corpus I were obtained with $n$ ranging within the interval $[3,7]$, and within the interval $[2,5]$ for Corpus II. It is surprising that being the MSI task more difficult with Corpus II, the results are similar or even much better. The explanation is that the $n$-gram models tipically need thousands of training samples to obtain good results, and we have very few (just 100 per style). Though in Corpus II the number of samples has not been numerically augmented, the number of running notes (see Section 5) has been increased by nearly 20 times. So, the number of $n$-grams that can be found increases tremendously and a much greater amount of data is available for building the models.

The experiments with MLPs shown in Table 2 are very preliminary, much more experimentation is needed to show the effectiveness of NNs for this task. For Corpus I we obtained, with a codification encoding of absolute pitch and relative duration, a classification error of $4 \%$, with a $95 \%$ confidence interval which intersects with the best error obtained for $n$-grams. Nevertheless, with a codification encoding of relative pitch and absolute duration, the MLP converged in a very poor classification rate (and thus we did not perform experiments with Corpus II with this encoding). For Corpus II the best classification error rate was $23.3 \%$, very far from the performance with $n$-grams. We think that fix-dimensional input NNs, such as MLPs, may not be adequate for this task, with great variability of length (the whole musical pieces range from 61 to 1825 notes).

\section{Conclusions and Future Trends}

The field of MSI is being recently explored, mostly in the field of multimedia databases, with potential applications to content-based retrieval. We have tried to face up this challenge with two different methodologies: $n$-gram Models and NNs. Excellent results were obtained with $n$-grams, whilst much worse results were obtained with MLPs. Nevertheless, the experiments with NNs are very preliminary, much more experimentation is needed to show the effectiveness of NNs for this task. For instance, we are studying other codification encoding which may be much more suitable for NNs. By other hand, for inmediate future 
work we are also considering to use classifiers based on recurrent NNs and hidden Markov models, which are capable of dealing with temporal sequences.

\section{Acknowledgements}

The authors wish to thank Diana Expósito for her help in obtaining the music representations and the experiments with NNs.

\section{References}

1. Pollastri, E., Simoncelli, G.: Classification of melodies by composer with hidden markov models. In: First International Conference on WEB Delivering of Music, pp. 88-95. IEEE Computer Press, Washington (2001)

2. de León, P.P., Iñesta, J.: Musical style classification from symbolic data: A twostyles case study. In: Wiil, U.K. (ed.) CMMR 2003. LNCS, vol. 2771, pp. 166-177. Springer, Heidelberg (2004)

3. Soltau, H., et al.: Recognition of music types. In: Proc. ICASSP 1998, pp. 11371140. Seattle, Washington (1998)

4. de la Higuera, C., Piat, F., Tantini, F.: Learning stochastic finite automata for musical style recognition. In: Conf. on Implementation and Application of Automata, pp. 345-346 (2005)

5. Cruz, P.P., Vidal, E., Pérez, J.C.: Musical style identification using grammatical inference: The encoding problem. In: Sanfeliu, A., Ruiz-Shulcloper, J. (eds.) CIARP 2003. LNCS, vol. 2905, pp. 375-382. Springer, Heidelberg (2003)

6. Cruz, P.P.: Técnicas de Reconocimiento de Formas Para el Modelado de Estilos Musicales. PhD thesis, Universidad Politécnica de Valencia (2004)

7. Cruz, P.P., Vidal, E.: Two grammatical inference applications in music processing. Applied Artificial Intelligence 22(1-2), 53-76 (2008)

8. Jelinek, F.: Statistical Methods for Speech Recognition. MIT Press, Cambridge (1998)

9. Katz, S.M.: Estimation of probabilities from sparse data for language model component of a speech recognizer. IEEE Trans. on ASSP 35(3), 400-401 (1987)

10. Clarkson, P., Rosenfeld, R.: Statistical language modeling using the CMUcambridge toolkit. In: Proc. Eurospeech 1997, vol. 5, pp. 2707-2710 (1997)

11. Witten, I.T., Bell, T.C.: The zero-frequency problem: Estimating the probabilities of novel events in adaptive text compression. IEEE Trans. on Information Theory 37(4), 1085-1094 (1991)

12. Rumelhart, D.E., Hinton, G.E., Williams, R.J.: Learning internal representations by error propagation. In: Rumelhart, D.E., McClelland, J.L. (eds.) PDP: Computational models of cognition and perception, I, pp. 319-362. MIT Press, Cambridge (1986)

13. Bishop, C.M.: Neural networks for pattern recognition. Oxford Univ. Press, Oxford (1995)

14. Zell, A., et al.: SNNS: Stuttgart Neural Network Simulator v. 4.2. Inst. Parallel and Distributed High Performance Systems, Univ. Stuttgart, Germany (1998) 
15. Wiggins, G.A.: A framework for the evaluation of music representation systems. Computer Music Journal 17(3), 31-42 (1993)

16. Cambouropoulos, E., Crawford, T., Iliopoulos, C.S.: Pattern processing in melodic sequences. Computers and the Humanities 35(1), 9-21 (2001)

17. Selfridge-Field, E.: Representing musical information for retrieval. In: SIGIR: Exploratory Workshop on Music Information Retrieval. ACM, Berkeley (1998)

18. Expósito Martín, D.: Detección automática de estilos musicales con redes neuronales. Master thesis, Facultad Informática, Univ. Politécnica de Valencia (2005) 